\title{
MAPPING THE NUCLEUS OF NGC1068 IN CO(2-1)
}

\author{
A.J. BAKER AND N.Z. SCOVILLE \\ California Institute of Technology \\ Astronomy 105-24, Pasadena, CA 91125
}

Molecular gas in active galactic nuclei (AGN) may play multiple roles: it can fuel a massive black hole, collimate jets, and/or obscure a broad-line region from most viewing angles. In order to understand the physical state and kinematics of molecular gas in AGN, we are using the Owens Valley Millimeter Array to map several nearby active galxies at high resolution in the $\mathrm{CO}(2-1)$ line. Our first target is NGC 1068, which hosts an obscured broad-line Seyfert nucleus as well as an extended starburst. We acquired data from 35 baselines out to $242 \mathrm{~m}$, with $10.4 \mathrm{~km} \mathrm{~s}^{-1}$ resolution. After calibration with MMA (Scoville et al. 1992), we subtracted a $23 \mathrm{mJy}$ nuclear continuum source from the visibility data and made CLEAN maps with AIPS. The resulting sensitivity in each line channel is $32 \mathrm{mJy}$ per $1.8^{\prime \prime} \times 1.5^{\prime \prime}$ robustly-weighted beam $\left(1^{\prime \prime}=70 \mathrm{pc}\right.$ at $\left.14.4 \mathrm{Mpc}\right)$.

Our integrated intensity map only weakly detects the spiral arms which dominate $\mathrm{CO}(1-0)$ maps, due to severe primary beam attenuation. However, there is a strong detection of molecular gas in the nucleus (Figure 1a). This structure has a FWZI linewidth of $380 \mathrm{~km} \mathrm{~s}^{-1}$, a peak $T_{\mathrm{b}}=9.4 \mathrm{~K}$, and a total $L_{\mathrm{CO}(2-1)}=5.5 \times 10^{7} \mathrm{~K} \mathrm{~km} \mathrm{~s}^{-1} \mathrm{pc}^{2}$. Two strong lobes lie to the east and west of the continuum source but do not trace the kinematic major axis, which the line-wing channel maps reveal to have $\mathrm{PA} \simeq 125^{\circ}$. This angle is similar to both the $\mathrm{PA}=135^{\circ} \mathrm{HCN}(1-0)$ kinematic major axis found by Tacconi et al. (1994) and the $\mathrm{PA}=113^{\circ}$ parsec-scale ionized gas disk found by Gallimore et al. (1997). Moving from the line wings to the line center, the red-and blue-shifted channel maps suggest symmetry about the "origin" (i.e. the continuum source). However, because none of the channel maps show reflection symmetry about the kinematic major axis, the molecular gas in the nucleus cannot be in a simple flat disk.

The kinematics may be consistent with inflow motions along a bar, as suggested by Tacconi et al. (1994), or with a discontinuous ring. However, they are also qualitatively consistent with a [nearly edge-on] warped disk. 

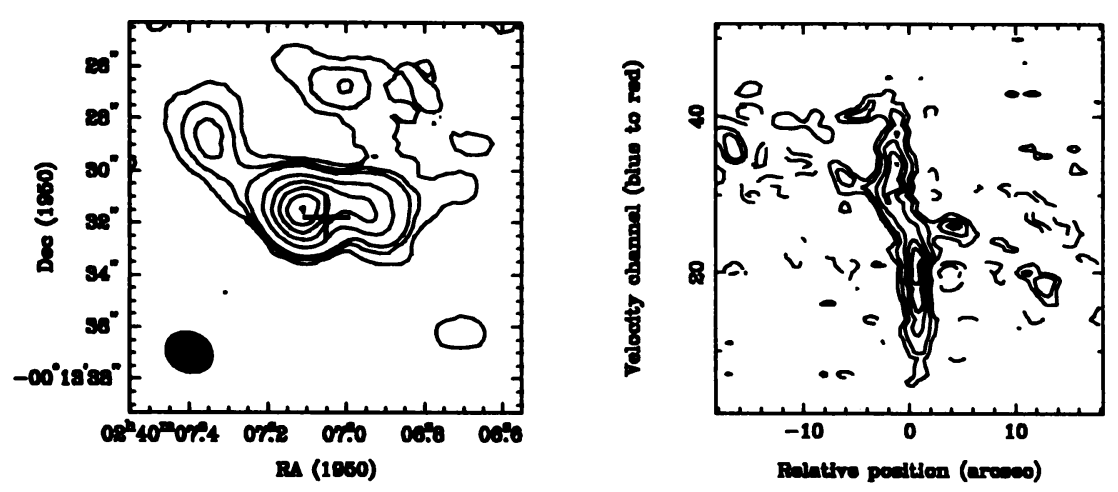

Figure 1. (a) Integrated intensity in $\mathrm{CO}(2-1)$. Contours are $\pm 1, \pm 2, \pm 3 n \times 5.5$ $\mathrm{Jy} \mathrm{beam}^{-1} \mathrm{~km} \mathrm{~s}^{-1}$. Cross marks position of the continuum source. (b) Position-velocity cut through the nucleus along $\mathrm{PA}=125^{\circ}$. Contours are $\pm 1, \pm 2 n \times 60 \mathrm{mJy} \mathrm{beam}^{-1}$.

In this picture, the kink at systemic velocity in a position-velocity cut along the kinematic major axis (Figure 1b) is due to the predominance of linewing gas in circular rotation at small radii. We can explain the difference in $T_{b}$ between east and west by a slight tilt of the disk (in addition to a warp), which lets us see one disk surface that is more directly heated by the central engine than the other. The sense of this tilt would be consistent with the observed extinction of emission from the galaxy's narrow-line region (Macchetto et al. 1994).

We have also obtained observations of NGC 1068 in the $\mathrm{CO}(1-0)$ line. At the $3.6^{\prime \prime} \times 3.5^{\prime \prime}$ resolution of our $\mathrm{CO}(1-0)$ map, we find a nuclear $\mathrm{CO}(2-$ $1) /(1-0)$ ratio [in $\mathrm{K}]$ of $\sim 1.9$, indicating that the gas we see has high excitation temperature and low optical depth. We also infer (for a Galac ${ }^{+i}$ c conversion factor) at most a total nuclear $M_{\mathrm{H}_{2}}=8.3 \times 10^{7} \mathrm{M}_{\odot}$. This is lower than the dynamical mass within $90 \mathrm{pc}$ of the nucleus, $M_{\mathrm{dyn}}=6.5 \times 10^{8} \mathrm{M}_{\odot}$, which we estimate from the $\mathrm{CO}(2-1)$ line wings assuming they come from an inner, relatively flat part of the nuclear structure.

\section{References}

Gallimore, J.F., Baum, S.A., and O'Dea, C.P. 1997, Nature, in press

Macchetto, F., Capetti, A., Sparks, W.B., Axon, D.J., and Boksenberg, A. 1994, ApJL, 435, L15

Scoville, N.Z., Carlstrom, J.E., Chandler, C.J., Phillips, J.A., Scott, S.L., Tilanus, R.P., and Wang, Z. 1992, PASP, 105, 1482

Tacconi, L.J., Genzel, R., Blietz, M., Cameron, M., Harris, A.I., and Madden, S. 1994, ApJL, 426, L27 